\title{
CASE OF THE MONTH
}

\section{Ultrasound and MRI features of connatal cysts: clinicoradiological differentiation from other supratentorial periventricular cystic lesions}

Z-Y J TAN, MBBS/B, Med Sci, P NAIDOO, MBBS, FRANZCR and N KENNING, MBChB, FRANZCR

Southern Health, 246 Clayton Road, Clayton 3168, Victoria, Australia

Received 2 August 2009

Accepted 24 August 2009

DOI: $10.1259 / \mathrm{bjr} / 10458905$

(1) 2010 The British Institute of Radiology

A 2-week-old neonate, born at 39 weeks via an uncomplicated vaginal delivery following a normal pregnancy, presented with a febrile illness suspected to be meningitis. A cranial ultrasound scan was performed (Figure 1).

As the patient had a persistent febrile illness, MRI was performed for investigation of suspected meningoencephalitis (Figure 2).

What is your diagnosis?

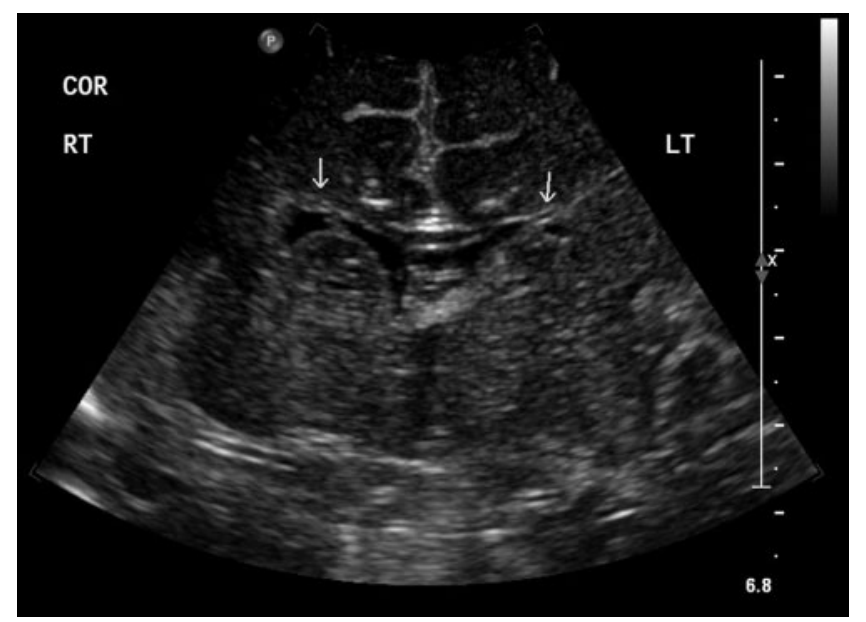

Figure 1. Coronal ultrasound image.

Address correspondence to: Z-Y J Tan, Southern Health, 246 Clayton Road, Clayton 3168, Victoria, Australia. E-mail: zhi_yie@ yahoo.com.au 


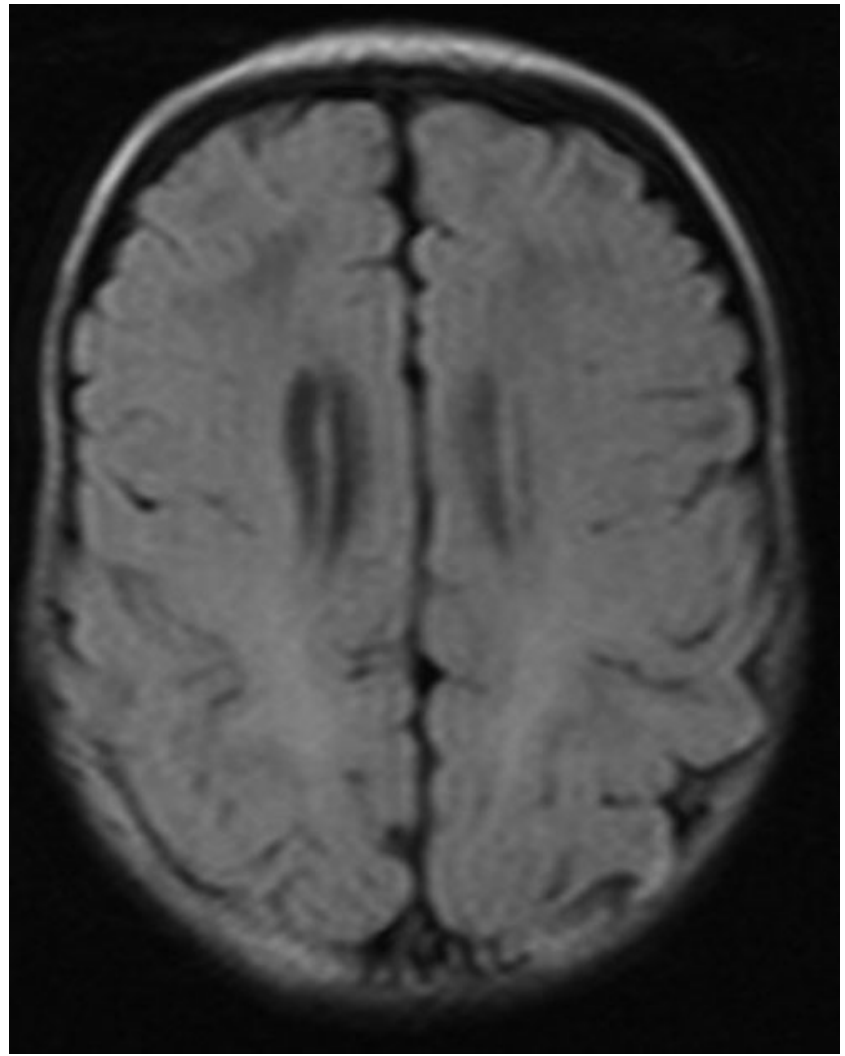

(a)

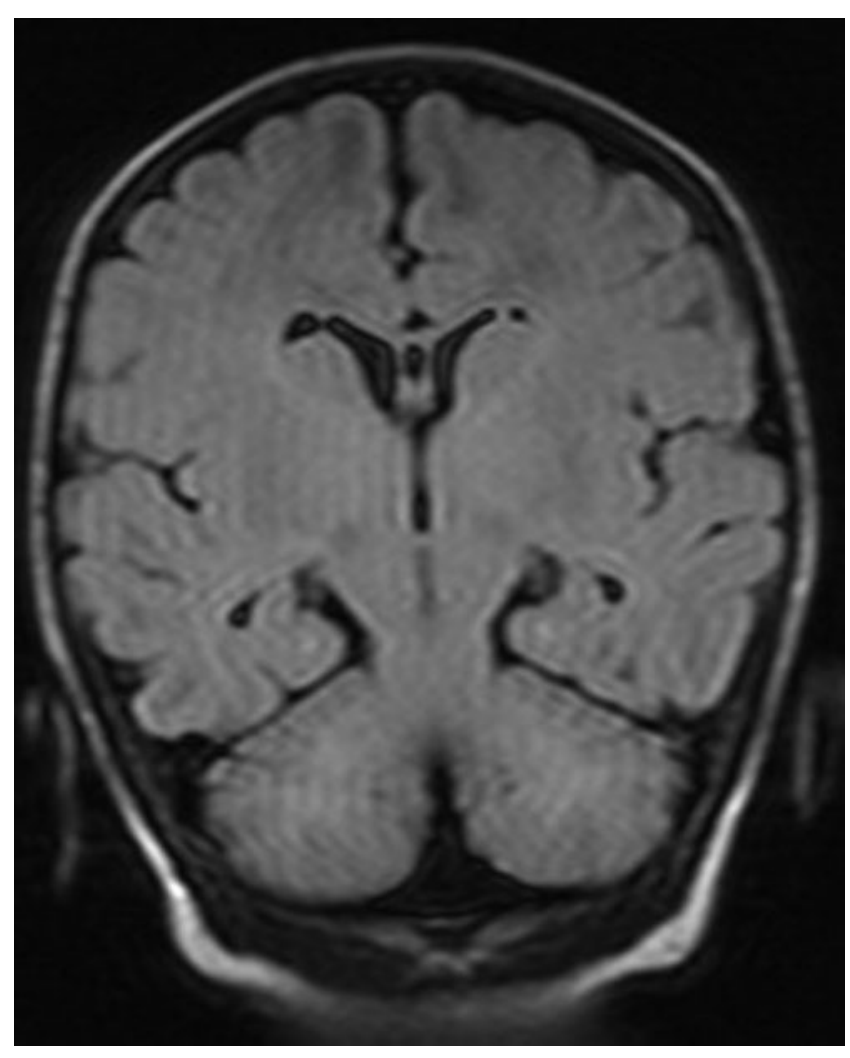

(c)

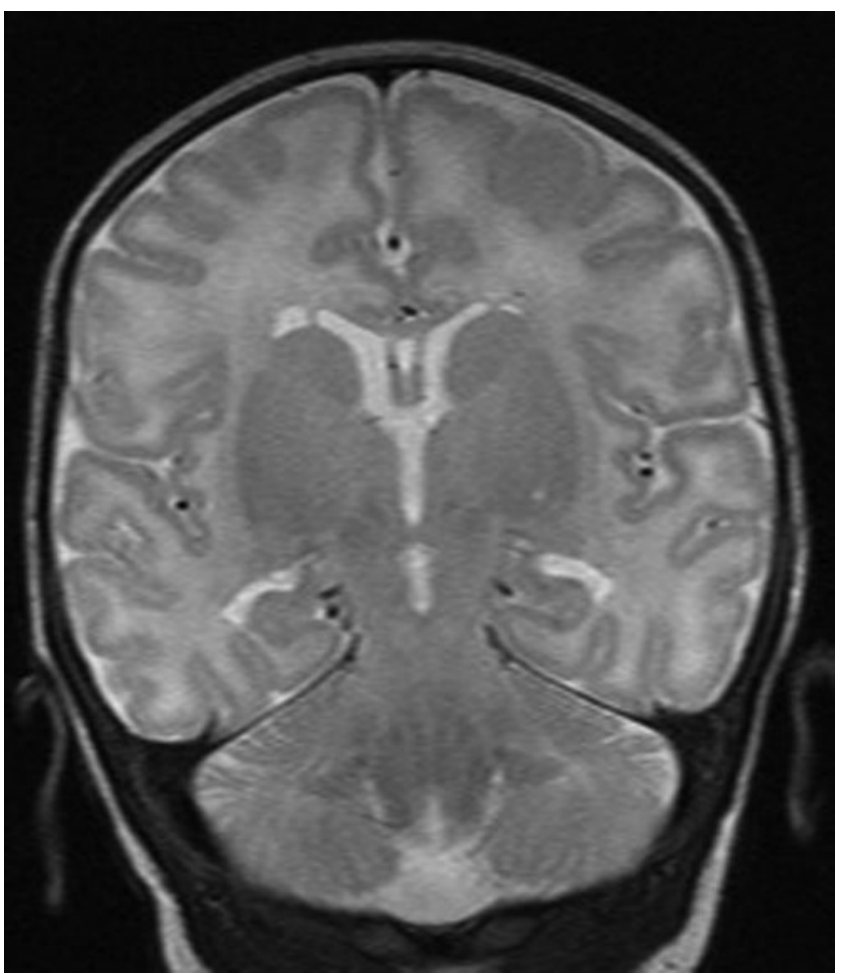

(b)

onal T2-weighted (b) and coronal FLAIR (c) MR images performed to investigate suspected meningoencephalitis. 


\section{Findings}

Cranial ultrasound demonstrated septations related to bilateral lateral ventricles, producing elongated cystic lesions that paralleled the frontal horns and bodies of the lateral ventricles. There was no associated germinal matrix haemorrhage, ventricular dilatation or periventricular parenchymal abnormality. The parenchymal volume was subjectively normal.

MRI confirmed the presence of periventricular cystic lesions with contents isointense to cerebrospinal fluid. Importantly, there was no gliosis, volume loss, or intraparenchymal or subependymal haemorrhage. There was no evidence of intracranial infection. The clinical features and imaging findings were therefore felt to be consistent with bilateral connatal cysts or "coarctation of the lateral ventricles".

\section{Discussion}

Periventricular cysts are a common finding in neonatal cranial imaging. The differential diagnoses of supratentorial periventricular cysts are numerous, and include connatal cysts, subependymal cysts, porencephalic cysts and cystic periventricular leukomalacia (Figure 3).

Connatal cysts are rare, with an incidence of $0.7-1.05 \%$ in low-birth-weight infants [1]. This incidence in term infants is unknown, as no routine cranial ultrasound is performed. Connatal cysts are considered normal anatomical variants that involve the walls of the lateral ventricles and lie at or just below the superolateral aspect of the frontal horns or body of the lateral ventricles, anterior to the foramen of Monro. There is no association with antenatal or perinatal events. The natural history is that of resolution at 1-2 months corrected age [1], with no adverse neurological outcome.

In contrast to connatal cysts, subependymal cysts are typically located below the external angles of the lateral ventricles and posterior to the foramen of Monro. They are often tear shaped and range from $2 \mathrm{~mm}$ to $11 \mathrm{~mm}$ in size [2]. Subependymal cysts can be divided into two types: acquired (secondary to haemorrhage, hypoxia-ischaemia or infection) and congenital (resulting from germinolysis). They frequently present following Grade 1 germinal matrix haemorrhage, which is associated with prematurity. Typically, subependymal cysts have a good prognosis.

In comparison, porencephalic cysts are late sequelae of a destructive process (e.g. intraparenchymal haemorrhage, infection or surgery) and have an overall prevalence of $2.5 \%$ in children with perinatal brain injury [3]. These cysts are usually (i) large, (ii) associated with parenchymal volume loss, (iii) multiple and noncommunicating and (iv) able to communicate with the ventricular system or subarachnoid space. They are more commonly unilateral or asymmetric, and seldom disappear over time. The prognosis is dependent on the size and extent of the initial insult, and is often associated with major intellectual-neurological deficits and even death.

Cystic periventricular leukomalacia may develop into porencephalic cysts. Watershed areas sensitive to ischaemia - at the peritrigonal area and anterior and lateral to the frontal horns, or above the external angles of the lateral ventricles - are the typical locations for periventricular leukomalacia. Risk factors for white matter necrosis include hypotension, hypocarbia, infection, prematurity, asphyxia, sepsis, multiple gestation, respiratory distress and maternal haemorrhage. Volume loss of the involved white matter often develops, producing ventricular enlargement and/or cerebral atrophy. The overall prognosis for cystic periventricular leukomalacia is poor; in particular, the presence of bilateral large $(>10 \mathrm{~mm})$ parieto-occipital cysts is highly predictive for the development of cerebral palsy [1].

For porencephalic cysts and cystic periventricular leukomalacia, MRI in particular is likely to demonstrate gliosis, volume loss, and, in the former, evidence of haemosiderin staining.

\section{Conclusions}

Given the wide differences in prognoses, the precise diagnosis of supratentorial periventricular cystic lesions is

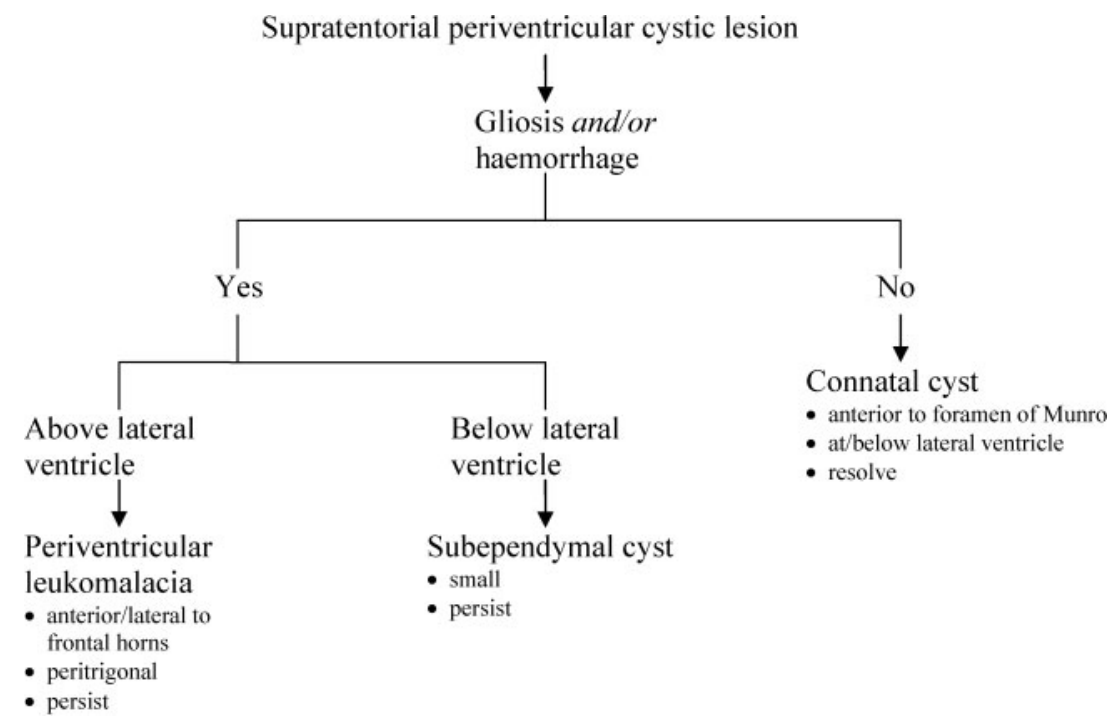

Figure 3. Key imaging features in determining the diagnosis of supratentorial periventricular cystic lesions. 
imperative. Clinical and imaging features are paramount in determining the cause and aetiology of these cysts.

\section{References}

1. Pal BR, Preston PR, Morgan MEI, Rushton DI, Durbin GM. Frontal horn thin walled cysts in preterm neonates are benign. Arch Dis Child Fetal Neonatal Ed 2001;85:F187-93.
2. Larcos G, Gruenewald SM, Lui K. Neonatal subependymal cysts detected by sonography: prevalence, sonographic findings, and clinical significance. AJR Am J Roentgenol 1994;162:953-6.

3. Epelman M, Daneman A, Blaser SI, Ortiz-Neira C, Konen O, et al. Differential diagnosis of intracranial cystic lesions at head us: correlation with ct and $\mathrm{mr}$ imaging. Radiographics 2006;26:173-96. 